\title{
Changes in metamorphopsia in daily life after successful epiretinal membrane surgery and correlation with M-CHARTS score
}

This article was published in the following Dove Press journal:

Clinical Ophthalmology

4 February 2015

Number of times this article has been viewed

\author{
Takamasa Kinoshita ${ }^{1,2}$ \\ Hiroko Imaizumi' \\ Hirotomo Miyamoto' \\ Utako Okushiba' \\ Yuki Hayashi² \\ Takashi Katome ${ }^{2}$ \\ Yoshinori Mitamura ${ }^{2}$ \\ 'Department of Ophthalmology, \\ Sapporo City General Hospital, \\ Sapporo, Japan; ${ }^{2}$ Department of \\ Ophthalmology, Institute of Health \\ Biosciences, The University of \\ Tokushima Graduate School, \\ Tokushima, Japan
}

\begin{abstract}
Purpose: To determine the correlation between the changes in metamorphopsia in daily life environment and the M-CHARTS scores after epiretinal membrane (ERM) removal, and to determine the criterion for determining whether clinically significant changes in the metamorphopsia score have occurred in M-CHARTS.
\end{abstract}

Methods: We studied 65 eyes undergoing vitrectomy for unilateral ERM. Self-administered questionnaires were used to examine the metamorphopsia in their daily life. The degree of metamorphopsia was determined by M-CHARTS. The receiver operating characteristic curve was used to determine the best predictor of the changes in metamorphopsia in daily life. To determine the reproducibility of the M-CHARTS score, another set of 56 eyes with ERM was tested twice on two different days.

Results: The postoperative changes in the logarithm of the M-CHARTS score was defined as M2-value. The area under the receiver operating characteristic curve for the M2-value as a predictor of the changes in metamorphopsia in daily life was larger than area under the receiver operating characteristic curve obtained for any other parameter. The optimal cutoff value was -0.4 . The 95\% limits of agreement between test and retest measurements had a reproducibility of \pm 0.3 logarithm of the M-CHARTS score. Taking into account not only the reproducibility but also the consistency with the subjective changes, we determined the criterion for clinically significant changes in the M-CHARTS scores as a change of the M2-value by $\geq 0.4$.

Conclusion: Evaluating the changes in the M-CHARTS scores in logarithmic form is favorable not only theoretically but also from the perspective of consistency with the subjective changes.

Keywords: metamorphopsia score, ERM, criterion, vitrectomy

\section{Introduction}

Metamorphopsia is the major cause of a decrease in vision in patients with an idiopathic epiretinal membrane (ERM). Amsler charts are commonly used to detect the metamorphopsia, ${ }^{1}$ but it is difficult to quantify the degree of the metamorphopsia with these charts. To overcome this limitation, Matsumoto et al developed a chart, called M-CHARTS (Inami Co., Tokyo, Japan), that can quantify the severity of the metamorphopsia. These charts have been used to quantify the severity of metamorphopsia associated with different macular diseases. ${ }^{2-12}$ Importantly, the M-CHARTS scores have been shown to be significantly correlated with the degree of metamorphopsia quantified by a questionnaire. ${ }^{7}$

It is important to evaluate the degree of metamorphopsia in terms of daily living to determine the quality of life of the patients. ${ }^{5,13}$ However, only a limited number of studies have prospectively investigated the presence or absence of metamorphopsia 
in daily life situations after ERM surgery and determined whether there is a correlation between the presence of metamorphopsia or the postoperative changes in the degree of metamorphopsia and the M-CHARTS scores. In addition, there are no accepted criteria established for determining whether clinically significant changes in the metamorphopsia score have occurred.

Thus, the purpose of this study was to determine the presence or absence of metamorphopsia in daily life after ERM surgery, and to determine the correlation between the presence of metamorphopsia or the changes in the degree of metamorphopsia and the M-CHARTS scores. We also determined the cutoff value that determined that clinically significant changes in the M-CHARTS score had occurred after ERM surgery.

\section{Materials and methods}

The procedures used conformed to the tenets of the Declaration of Helsinki, and an informed consent was obtained from all of the subjects after the procedures to be used had been fully explained. An approval was obtained from the Institutional Review Board of Sapporo City General Hospital and Tokushima University Hospital to perform this study.

This study consisted of two parts. Part 1 was undertaken to determine the time course of the presence or absence of metamorphopsia under daily life conditions after the ERM surgery. In addition, we determined the correlation between the presence of metamorphopsia or the changes in the degree of metamorphopsia in daily life and the M-CHARTS scores. The changes in the degree of metamorphopsia in daily life were determined by a questionnaire. Then, we calculated the correlation between the changes in the degree of metamorphopsia and the M-CHARTS scores. This study was conducted as a part of our earlier study. ${ }^{9}$

Part 2 consisted of determining the reproducibility of the M-CHARTS scores. Based on the results of two parts of the study, we determined the criterion for assessing whether clinically significant changes in the M-CHARTS scores had occurred after the ERM surgery.

\section{Part I}

\section{Patients}

Part 1 was a prospective, consecutive case study of 65 eyes of 65 patients with metamorphopsia who underwent vitrectomy with idiopathic ERM removal. The patients were examined and operated on at the Sapporo City General Hospital between April 2008 and November 2011. One hundred and forty-nine consecutive eyes of 145 patients underwent ERM surgery by a single surgeon (TK). Eighty-four eyes were excluded; 15 eyes because of an absence of preoperative metamorphopsia, eight eyes because of bilateral idiopathic ERM, 34 eyes because the ERM was secondary to retinal tears, retinal detachment, retinal vascular diseases and uveitis, six eyes because of the presence of other macular diseases including age-related macular degeneration and central serous chorioretinopathy, four eyes because the bestcorrected visual acuity (BCVA) was less than 1.0 logarithm of the minimum angle of resolution ( $\log$ MAR) units, nine eyes because of previous intraocular surgery except for uncomplicated cataract surgery, and eight eyes because of moderate or severe cataract that affected the BCVA. In the end, 65 eyes of 65 patients were studied in Part 1.

\section{Ophthalmic examinations}

The ophthalmic examinations including measurements of the BCVA, applanation tonometry, slit-lamp biomicroscopy, indirect ophthalmoscopy, fundus photography, spectral-domain optical coherence tomography ([SD-OCT] 3D OCT 1000; Topcon Co., Tokyo, Japan), M-CHARTS measurements, and answering a questionnaire concerning the metamorphopsia in daily life were performed at the baseline, and at 1, 3, 6, 9, and 12 months postoperatively. All baseline data were obtained within 2 months of the surgery.

\section{SD-OCT}

The SD-OCT protocol used was described in detail in our earlier study. ${ }^{9}$ Briefly, cross sectional scan images of $6 \mathrm{~mm}$ length through the fovea and the $3 \mathrm{D}$ scan protocol, which was a raster scan composed of $256 \times 256$ axial scans covering a $6 \times 6 \mathrm{~mm}$ macular region were obtained for each eye. The central foveal thickness (CFT) and the center volume ([CV] area within a $1 \mathrm{~mm}$ diameter circle) were analyzed at baseline and 12 months after the surgery.

\section{Surgical procedures}

In Part 1, the main indication of ERM surgery was the patient's visual disabilities caused by the decrease in the visual acuity or metamorphopsia and the patient's consent. All surgeries were performed by a single surgeon (TK) using 25 gauge transconjunctival pars plana vitrectomy with ERM removal. Phacoemulsification and intraocular lens implantation were performed on all phakic cases. Six cases were already pseudophakic. After successful removal of the ERM, the internal limiting membrane was peeled with the assistance of triamcinolone acetonide in all cases. No other dye was used. In eleven cases with severe distortion of the retina, air-fluid exchange and postoperative facedown positioning for half a day were performed. An 
iatrogenic peripheral retinal tear developed in one eye and was successfully treated with endolaser photocoagulation.

\section{Self-administered questionnaires}

The questionnaires were self-administered and were used to determine whether metamorphopsia was present in their daily life. The questionnaire included the following three questions (Figure 1).

Subjects were instructed to examine their metamorphopsia in their daily life binocularly and uniocularly with their affected eyes. They were informed that their answers would not influence the ongoing ophthalmic examinations or treatments.

\section{Quantification of metamorphopsia}

The severity of the metamorphopsia was quantified with the M-CHARTS (Figure 2). The M-CHARTS consist of a single solid line and 19 dotted lines. The dot intervals ranged from $0.2^{\circ}$ to $2.0^{\circ}$ when viewed at $30 \mathrm{~cm}$. In patients with metamorphopsia, a straight solid line is reported to appear curved or irregular. If the solid line is replaced by a dotted line and the dot interval is increased from fine to coarse, the irregularity of the line gradually decreases until the dotted line appears straight. The minimum angle of the dots that appeared straight was taken to be the metamorphopsia score in degrees. ${ }^{2}$ The score for metamorphopsia for horizontal lines (MH) and metamorphopsia for vertical lines (MV) was measured separately. The MH and MV scores in normal eyes without metamorphopsia were 0 . The examiners who administered the M-CHARTS tests were experienced orthoptists who were masked to the clinical findings. We used a value of 2.0 for a metamorphopsia score $>2.0$ for the statistical analyses.

\section{Determining the correlation between the change in the degree of metamorphopsia in daily life and the M-CHARTS scores}

To determine the M-CHARTS score that indicated a significant change in the degree of metamorphopsia in daily life, we calculated the optimal cutoff value of the M-CHARTS score by the receiver operating characteristic (ROC) curve analysis. The area under the ROC curve (AUC) represents the overall diagnostic accuracy of a test. A test with perfect discrimination has an ROC curve that passes through the upper-left corner (100\% sensitivity and $100 \%$ specificity; AUC 1.0), whereas a test with no discrimination has a $45^{\circ}$ ROC curve (AUC 0.5). The AUC was determined for each variable obtained from the M-CHARTS scores to identify the predictors of the presence of an improvement or worsening in the degree of metamorphopsia in daily life (Table 1).

M-CHARTS scores are recorded in visual degree units which is similar to that for visual acuities (arc minutes). The visual acuities are usually converted to the logMAR units for statistical analyses, and similarly, the changes in the M-CHARTS scores were evaluated in logarithm of metamorphopsia (logMET) scores. For example, a change from 0.4 to 0.2 in the M-CHARTS score represents a $50 \%$ improvement in visual angle, whereas a change from 2.0 to 1.8 in the M-CHARTS score represents only a $10 \%$ improvement. But if these are expressed in logMET scores, the change in $\log$ MET score is -0.301 in the former case, and -0.046 in the latter case. A decrease or increase in the logMET scores represents the same rate of decrease or increase in the visual angle. For example, a decrease of $0.3 \log$ MET score is a change that halves the visual angles.

The changes in the M-CHARTS scores were defined as the postoperative value at 12 months subtracted from the baseline value. The following six possible predictors for the change in the degree of metamorphopsia in daily life were examined:

1) changes in the $\mathrm{MH}$ scores $\left(\mathrm{MH}_{\text {post }}-\mathrm{MH}_{\text {pre }}\right)$;

2) changes in the MV scores $\left(\mathrm{MV}_{\text {post }}-\mathrm{MV}_{\text {pre }}\right)$;

3) either the $\left(\mathrm{MH}_{\text {post }}-\mathrm{MH}_{\text {pre }}\right)$ or the $\left(\mathrm{MV}_{\text {post }}-\mathrm{MV}_{\text {pre }}\right)$, whichever of these absolute values was larger was used and called M1-value;

1. Do you have distorted vision when you see things around you with both eyes in your daily life? Yes or No?

2. Do you have distorted vision when you see things around you after covering your unaffected eye in your daily life? Yes or No?

3. How would you rate the change in the degree of distorted vision after the surgery to that you had before? Improved, Unchanged, or Worsened?

Figure I Self-administered questionnaire used in the study.

Notes: Questions I and 2 were asked at each visit, and Question 3 was asked only at 12 months after the surgery. 


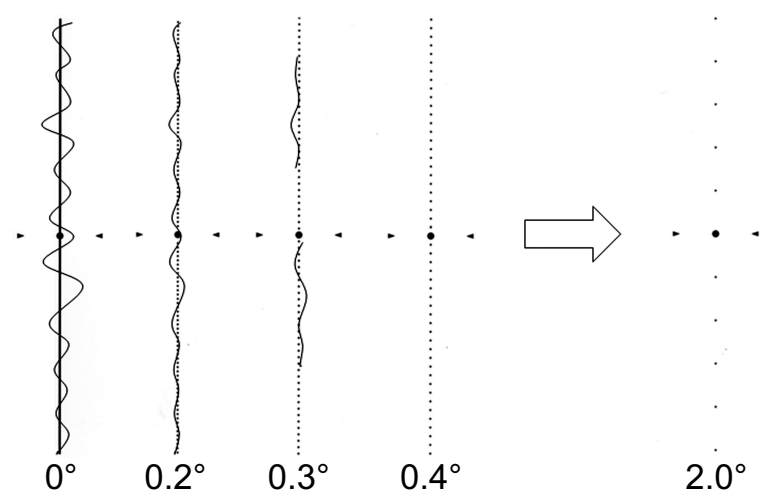

Figure 2 Method of determining the metamorphopsia score using M-CHARTS. Notes: The minimum angle of the dots that appeared straight was taken to be the metamorphopsia score. In this case, metamorphopsia for vertical lines (MV) is 0.4 .

4) changes in the logarithm of $\mathrm{MH}$ score $\left(\log \mathrm{MH}_{\text {post }}{ }^{-}\right.$ $\left.\log \mathrm{MH}_{\text {pre }}\right)$;

5) changes in the logarithm of MV score $\left(\log \mathrm{MV}_{\text {post }}{ }^{-}\right.$ $\left.\log \mathrm{MV}_{\mathrm{pre}}\right)$;

6) either $\left(\log \mathrm{MH}_{\text {post }}-\log \mathrm{MH}_{\mathrm{pre}}\right)$ or $\left(\log \mathrm{MV}_{\text {post }}-\log \mathrm{MV}_{\mathrm{pre}}\right)$, whichever of these absolute values was larger was used and called M2-value.

We used a value of 0.1 for a metamorphopsia score to calculate the $\log \mathrm{MH}$ or $\log \mathrm{MV}$ score when the metamorphopsia score was 0 . Examples of these variables are shown in Table 2.

\section{Part 2}

The purpose of this part was to determine the reproducibility of the M-CHARTS scores. This was used to determine whether significant changes in the visual acuity had occurred. ${ }^{14-16}$

\section{Patients}

Part 2 was a prospective, consecutive case series of 56 eyes of 56 patients who had metamorphopsia and underwent vitrectomy and ERM removal at the Sapporo City General Hospital and Tokushima University Hospital between

Table I A contingency table of diagnostic tests

\begin{tabular}{llll}
\hline Test & Disease & & Total \\
\cline { 2 - 3 } & Present & Absent & \\
\hline Positive & TP & FP & TP + FP \\
Negative & FN & TN & FN + TN \\
Total & TP + FN & FP + TN & \\
\hline
\end{tabular}

Notes: "Disease" in this study corresponds to the presence of an improvement or worsening in the degree of metamorphopsia in daily life after epiretinal membrane surgery.

Abbreviations: TP, true positive response; FN, false negative response; FP, false positive response; TN, true negative response.
May 2012 and April 2013. The exclusion criteria were the same as those of Part 1.

\section{Determining the reproducibility of the M-CHARTS scores}

The reproducibility of the M-CHARTS scores was measured by determining the $95 \%$ limits of agreement between test and retest measurements before surgery. A $95 \%$ limit of agreement was determined as the mean difference $\pm 1.96 \times$ standard deviation of the differences as reported. ${ }^{17}$ Test and retest were performed on different days within a month. Cases whose degree of metamorphopsia in their daily life had improved or worsened between test and retest were excluded because the degree of metamorphopsia had to be stable between test and retest for examining the reproducibility. We calculated the reproducibility of the $\log \mathrm{MH}$ and $\log \mathrm{MV}$ scores as well as the absolute MH and MV scores. The M-CHARTS examinations were performed by six orthoptists at two institutions. The test and retest were performed by different examiners who were randomly selected.

Then, we assessed the criterion for a significant change in the M-CHARTS score by comparing the reproducibility of the M-CHARTS scores with the cutoff value obtained from the ROC analysis in Part 1.

\section{Statistical analyses}

Statistical analyses were carried out with the StatMate III (ATMS, Tokyo, Japan) and SPSS statistics version 19 (IBM Corporation, Armonk, NY, USA). The significance of the differences in the MH or MV scores at two visits or between test and retest were determined with the Wilcoxon signedrank test. The significance of the differences in the baseline demographic data between the cases in Part 1 and Part 2 and the significance of difference in the CFT and CV between two groups were determined by Mann-Whitney $U$-test. The correlation in the MH or MV scores between test and retest was analyzed using Spearman's rank correlation coefficient. A two-sided $P$-value $<0.05$ was considered statistically significant.

\section{Results \\ Baseline demographic data}

The baseline demographic data of all of the patients are shown in Table 3. There were no significant differences in any of the demographic data between the eyes in Part 1 and Part 2. All 65 patients in Part 1 had metamorphopsia when examining their surroundings with their affected eyes. Among these, eleven $(16.9 \%)$ patients also had metamorphopsia when they 
Table 2 Examples of concerning explanation variables

\begin{tabular}{|c|c|c|c|c|}
\hline & $\mathbf{M H}$ & $\log \mathrm{MH}$ & MV & $\log M V$ \\
\hline Baseline & 1.5 & 0.176 & 1.0 & 0 \\
\hline 12 months & 0.5 & -0.301 & 0.8 & -0.096 \\
\hline \multirow[t]{2}{*}{ Change } & -1.0 & -0.477 & -0.2 & -0.096 \\
\hline & $M H_{\text {post }}-\mathrm{MH}_{\text {pre }}$ & $\log \mathrm{MH}_{\text {post }}-\log \mathrm{MH}_{\text {pre }}$ & $M V_{\text {post }}-M V_{\text {pre }}$ & $\log \mathrm{MH}_{\text {post }}-\log \mathrm{MH}_{\text {pre }}$ \\
\hline
\end{tabular}

Notes: When the $\mathrm{MH}$ score and the MV score were I.5 and I.0 at baseline, and 0.5 and 0.8 at 12 months after the surgery respectively, MI-value was $-\mathrm{I} .0$ and $M 2$-value was -0.477 . $M I$-value $=$ either $\left(\mathrm{MH}_{\text {post }}-\mathrm{MH}_{\text {pre }}\right)$ or $\left(\mathrm{MV}_{\text {post }}-\mathrm{MV}_{\text {pre }}\right)$ whichever of these absolute value was larger was used. $M 2$-value $=$ either $\left(\right.$ logMH $\left.\mathrm{H}_{\text {post }}-\mathrm{log} M \mathrm{MH}_{\text {pre }}\right)$ or $\left(\log M V_{\text {post }}-\log M V_{\text {pre }}\right)$ whichever of these absolute value was larger was used.

Abbreviations: $\mathrm{MH}$, metamorphopsia for horizontal lines; $\mathrm{MV}$, metamorphopsia for vertical lines; logMV, logarithm of $\mathrm{MV}$ score; log MH, logarithm of $\mathrm{MH}$ score.

looked at things binocularly. Fifteen eyes were excluded from the study because of an absence of metamorphopsia in their daily life. In these eyes, the highest MH score was 0.4 and the MV score was 0.3 .

\section{Changes in BCVA, the mean metamorphopsia scores, and the presence of metamorphopsia in daily life}

The mean \pm standard error of the mean (SEM) of the BCVA improved significantly from $0.33 \pm 0.02 \log$ MAR units before surgery to $0.09 \pm 0.02 \log$ MAR units at 12 months after the surgery $(P<0.001)$.

The time course of the changes in the relative number of patients without metamorphopsia, and the mean metamorphopsia scores are shown in Figure 3. The MH score improved significantly from $1.05 \pm 0.08$ before surgery to $0.38 \pm 0.06$ at 12 months after surgery $(P<0.001)$. The mean MH score improved rapidly during the first 3 months after surgery and then more slowly until 12 months. The MV score improved significantly from $0.89 \pm 0.07$ before surgery to $0.41 \pm 0.06$ at 12 months after surgery $(P<0.001)$. The mean MV score improved rapidly during the first month after surgery and then more slowly until 6 months when it reached a plateau.

The percentage of patients who did not have metamorphopsia increased as the metamorphopsia scores decreased until finally 23 (35.4\%) of 65 patients had no metamorphopsia in their daily life at 12 months (Figure 3). A complete normalization of the M-CHARTS scores for both the MH and
MV scores was achieved in $18(27.7 \%)$ patients at 12 months. The highest M-CHARTS score in those who reported no metamorphopsia postoperatively was 0.3 for both the $\mathrm{MH}$ and MV scores at 12 months after surgery. All patients with metamorphopsia score $\geq 0.4$ had subjective perception of metamorphopsia in their daily life at 12 months after the surgery.

From the questionnaire, 53 of 65 patients $(81.5 \%)$ with preoperative metamorphopsia in their daily life reported that their metamorphopsia had improved at 12 months from that at baseline. Twelve $(18.5 \%)$ patients reported it had not changed. None of the patients reported that their metamorphopsia had worsened.

\section{OCT parameters and metamorphopsia in daily life}

The mean CFT and CV in the 65 subject eyes at baseline were $414.4 \pm 16.8 \mu \mathrm{m}$ and $0.35 \pm 0.01 \mathrm{~mm}^{3}$ respectively. The CFT $(343.0 \pm 32.9 \mu \mathrm{m})$ and $\mathrm{CV}\left(0.28 \pm 0.01 \mathrm{~mm}^{3}\right)$ in the 15 eyes without metamorphopsia at baseline, which were excluded from the study, were significantly smaller than those in the tested eyes $(P<0.05$ for CFT, $P<0.01$ for CV). In the 65 subject eyes, there was no difference in the mean CFT and the $\mathrm{CV}$ at 12 months between the two groups with and without metamorphopsia in daily life at 12 months $(P=0.38$, $P=0.98$, respectively). There was no difference in the mean $\mathrm{CFT}$ and the $\mathrm{CV}$ at baseline between the two groups with and without metamorphopsia in daily life at 12 months. $(P=0.51$, $P=0.09$, respectively).

Table 3 Baseline demographic data of patients with ERM in Part I and Part 2

\begin{tabular}{llll}
\hline & Part I & Part 2 (test) & P-value \\
\hline Sex & 42 women/23 men & 27 women/29 men & 0.102 \\
Age (yrs), mean \pm SE (range) & $68.8 \pm 1.0(5 I-84)$ & $69.3 \pm I .1(53-91)$ & 0.729 \\
Preoperative BCVA (logMAR), mean \pm SE & $0.33 \pm 0.03$ & $0.40 \pm 0.03$ & 0.901 \\
Preoperative MH, mean \pm SE & $1.05 \pm 0.08$ & $0.88 \pm 0.06$ & 0.245 \\
Preoperative MV, mean \pm SE & $0.89 \pm 0.07$ & $0.74 \pm 0.05$ & 0.282 \\
\hline
\end{tabular}

Abbreviations: ERM, epiretinal membrane; SE, standard error; MH, metamorphopsia for horizontal lines; MV, metamorphopsia for vertical lines; yrs, years; BCVA, bestcorrected visual acuity; logMAR, logarithm of the minimum angle of resolution. 


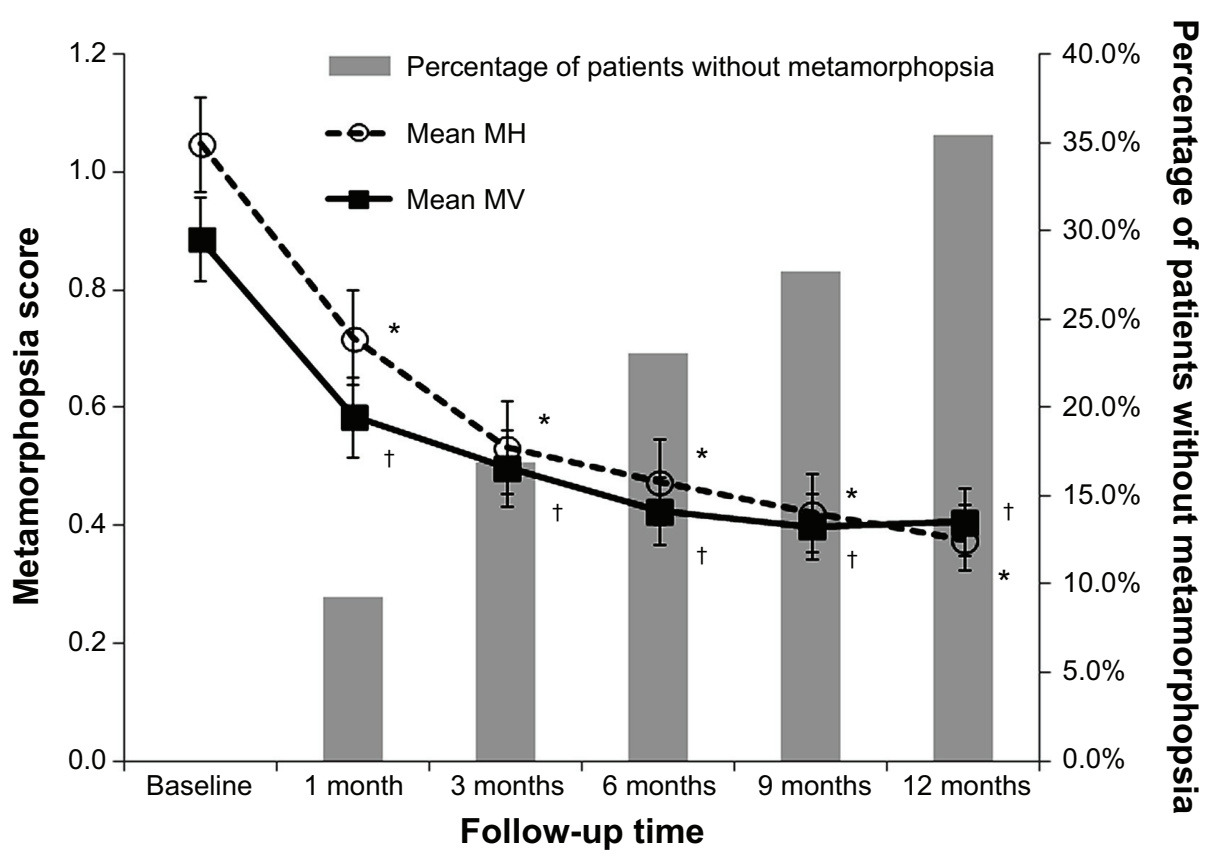

Figure 3 Changes in the percentage of patients without metamorphopsia and the mean metamorphopsia scores.

Notes: The percentage of patients without metamorphopsia increased as the metamorphopsia scores decreased until finally $35.4 \%$ of patients had no metamorphopsia in their daily life at 12 months. Error bars indicate standard error. ${ }^{*}$ tStatistically significant compared with the metamorphopsia score at baseline $(P<0.00 \mathrm{I})$, $\mathrm{n}=65$. Abbreviations: MV, metamorphopsia for vertical lines; $\mathrm{MH}$, metamorphopsia for horizontal lines.

\section{Correlation between the change in degree of metamorphopsia in daily life and M-CHARTS scores}

ROC curve analyses were performed to identify the predictors of improvement in the degree of metamorphopsia from the questionnaire (Figure 4). The AUC for all variables was significantly larger than that of an AUC of 0.5 ( $P<0.0001$; Figure 4, Table 4). The ROC curve with the largest AUC was obtained for the M2-value. The ROC plot was nearest the upper-left corner when the criterion was $<-0.375$ of the M2-value. When the cutoff value was set at -0.4 , the sensitivity was $86.8 \%$ and the specificity was $100 \%$.

\section{Reproducibility of the M-CHARTS scores}

Fifty-six eyes of 56 cases were studied in Part 2. The cases with MH or MV scores of 0 were excluded and 53 eyes were used to assess the reproducibility of the MH score and 52 eyes for the MV score. There was no difference in the MH, MV, $\log \mathrm{MH}$, or $\log \mathrm{MV}$ scores between the test and retest $(P=0.430$, $P=0.632, P=0.415, P=0.468$, respectively; Table 5). The testretest measurements of the $\mathrm{MH}, \mathrm{MV}, \log \mathrm{MH}$, or $\log \mathrm{MV}$ scores were significantly correlated $(r=0.824, P<0.001$; $r=0.876, P<0.001 ; r=0.824, P<0.001 ; r=0.876, P<0.001$; respectively). The $95 \%$ limits of agreement were from -0.491 to 0.510 for the $\mathrm{MH}$ score, from -0.402 to 0.417 for the
MV score, from -0.260 to 0.284 for the $\log M H$ score, and from -0.240 to 0.258 for the $\log \mathrm{MV}$ score. Reproducibility of $\log$ MET scores (ie, $\log \mathrm{MH}$ and $\log \mathrm{MV}$ ) are within \pm 0.3 (Table 5). Thus, detecting a clinically relevant change in the

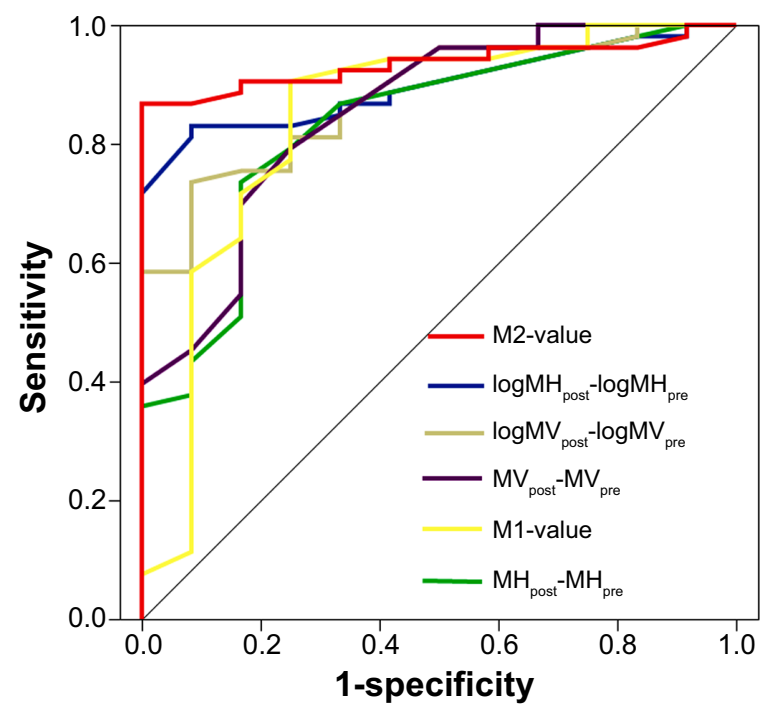

Figure 4 ROC curve for six variables to predict the change in the metamorphopsia in daily life.

Notes: The ROC curve with the largest AUC was obtained for the M2-value. $M I=$ either $\left(\mathrm{MH}_{\text {post }}-\mathrm{MH}_{\text {pre }}\right)$ or $\left(\mathrm{MV}_{\text {post }}-\mathrm{MV}_{\text {pre }}\right)$, whichever of these absolute values was larger was used. $M 2$-value = either $\left(\log M H_{\text {post }}-\log M H_{\text {pre }}\right)$ or $\left(\log M V_{\text {post }}-\log M V_{\text {pre }}\right)$, whichever of these absolute value was larger was used.

Abbreviations: ROC, receiver operating characteristic; AUC, area under the ROC curve; logMV, logarithm of MV score; logMH, logarithm of MH score; MV, metamorphopsia for vertical lines; $\mathrm{MH}$, metamorphopsia for horizontal lines. 
Table 4 Comparison of AUC for six variables

\begin{tabular}{|c|c|c|c|c|}
\hline & AUC (95\% Cl) & Optimal cutoff value & $P I^{*}$ & $\mathbf{P 2}^{\dagger}$ \\
\hline M2-value & $0.936(0.877-0.994)$ & $<-0.375$ & $<0.000$ I & \\
\hline $\log \mathrm{MH}_{\text {post }}-\log \mathrm{MH}_{\text {pre }}$ & $0.899(0.824-973)$ & $<-0.228$ & $<0.0001$ & 0.329 \\
\hline $\log M V_{\text {post }}-\log M V_{\text {pre }}$ & $0.88 \mathrm{I}(0.790-0.97 \mathrm{I})$ & $<-0.165$ & $<0.0001$ & 0.136 \\
\hline$M V_{\text {post }}-M V_{\text {pre }}$ & $0.852(0.733-0.972)$ & $<-0.250$ & $<0.000$ I & 0.095 \\
\hline MI-value & $0.842(0.693-0.991)$ & $<-0.250$ & $<0.0001$ & 0.170 \\
\hline $\mathrm{MH}_{\text {post }}-\mathrm{MH}_{\text {pre }}$ & $0.831(0.705-0.957)$ & $<-0.350$ & $<0.0001$ & 0.117 \\
\hline
\end{tabular}

Notes: The receiver operating characteristic (ROC) curve with the largest AUC was obtained for the M2-value. *Comparison with diagonal. tPairwise comparison with the ROC curve for M2-value. MI = either (MHpost-MHpre) or (MVpost-MVpre), whichever of these absolute values was larger was used. M2-value = either (logMHpostlogMHpre) or (logMV post-logMVpre), whichever of these absolute value was larger was used.

Abbreviations: AUC, area under the ROC curve; MH, metamorphopsia for horizontal lines; MV, metamorphopsia for vertical lines; logMV, logarithm of MV score; logMH, logarithm of $\mathrm{MH}$ score; $\mathrm{Cl}$, confidence interval.

M-CHARTS score after ERM surgery required a change of $\geq 0.3 \operatorname{logMET}$ score.

\section{Determining the criterion for clinically significant changes in the M-CHARTS scores after the ERM surgery}

A cutoff value of -0.4 for the M2-value obtained from the ROC analysis was not within the range of reproducibility of $\operatorname{logMET}$ scores which was within \pm 0.3 as described. Taking into account not only the reproducibility but also the consistency with the subjective changes in metamorphopsia, we determined the criterion for clinically significant changes in the M-CHARTS scores after ERM surgery as a change of the M2-value by $\geq 0.4$. From this criterion, the M-CHARTS score improved by $\geq 0.4 \mathrm{M} 2$-value in 46 eyes (70.8\%), remained unchanged in 14 eyes $(21.5 \%)$, and worsened in five eyes (7.7\%) at 12 months. The optimal cutoff values of the absolute values including the $\mathrm{MH}_{\text {post }}-\mathrm{MH}_{\text {pre }}$, the $M V_{\text {post }}-\mathrm{MV}_{\text {pre }}$, and the M1-value were all within the range of reproducibility of the absolute values of the M-CHARTS (Table 4, Table 5).

\section{Discussion}

In Part 1, 15 eyes were not included in the statistical analyses because of an absence of metamorphopsia in their daily life. The highest MH score in these 15 eyes was 0.4 and the highest MV score was 0.3 . For patients with a M-CHARTS score $\geq 0.5$, all reported that they had metamorphopsia in their daily life at the baseline. Similarly, all patients with a M-CHARTS score $\geq 0.4$ reported metamorphopsia in their daily life at 12 months after the surgery. Thus, a M-CHARTS score of 0.3 to 0.5 may be the threshold for detecting patients with metamorphopsia in their daily life. Arimura et al reported that $41.1 \%$ of ERM patients with a M-CHARTS score $<0.5$ had no distorted vision in their daily life, and that only $15.0 \%$ of ERM patients with a M-CHARTS score $\geq 0.5$ had no metamorphopsia. ${ }^{7}$ However, their patients tested their metamorphopsia binocularly.

Although the majority of patients with an ERM have metamorphopsia, there are cases in which the metamorphopsia resolves even if the ERM remains. This may be partly due to a shift in the dominant eye accompanied by suppression of the affected eye. This is similar to the shift in eye dominance in patients with macular diseases. ${ }^{18}$ Our patients were instructed to check metamorphopsia by covering their unaffected eyes to eliminate the effect of shifting of sighting dominance to determine whether metamorphopsia was present.

From the questionnaire, $35.4 \%$ of patients reported no metamorphopsia at 12 months. Eighty-one point five percent of patients reported that their metamorphopsia had improved, and $18.5 \%$ of patients reported it was unchanged. Wong et al reported that the rate of patients with moderate to severe metamorphopsia decreased from $80 \%$ preoperatively

Table 5 Reproducibility of M-CHARTS scores

\begin{tabular}{lllll}
\hline & MH & logMH & MV & logMV \\
\hline Test $($ mean \pm SD) & $0.888 \pm 0.493$ & $-0.126 \pm 0.269$ & $0.751 \pm 0.431$ & $-0.195 \pm 0.254$ \\
Retest $($ mean \pm SD) & $0.898 \pm 0.494$ & $-0.114 \pm 0.248$ & $0.7599 \pm 0.443$ & $-0.186 \pm 0.241$ \\
Difference (mean \pm SD) & $0.01 \pm 0.255$ & $0.012 \pm 0.138$ & $-0.182 \pm 0.241$ & $0.009 \pm 0.127$ \\
Reproducibility (minimum/maximum) & $-0.491 / 0.510$ & $-0.260 / 0.284$ & $-0.402 / 0.417$ & $-0.240 / 0.258$ \\
\hline
\end{tabular}

Notes: Reproducibility of each M-CHARTS score was calculated as the mean difference ( \pm 1.96 SD of the difference) between test and retest measurements. Reproducibility of logMET scores (logMH and logMV) are within \pm 0.3 .

Abbreviations: $\mathrm{MH}$, metamorphopsia for horizontal lines; MV, metamorphopsia for vertical lines; SD, standard deviation; logMV, logarithm of MV score; logMH, logarithm of $\mathrm{MH}$ score; logMET, logarithm of metamorphopsia. 
to $30 \%$ postoperatively. ${ }^{19}$ Bouwens et al reported that metamorphopsia improved in $62 \%$ of the patients, was unchanged in $21 \%$, and worsened in $17 \%$ at 5 to 6 years after ERM removal. ${ }^{20}$ Our results are compatible with these findings although we cannot simply compare the results because of the different methodologies.

The rates of metamorphopsia resolution improved continuously throughout the 12 months after the surgery. On the other hand, the mean $\mathrm{MH}$ score improved rapidly during the first 3 months after the surgery and then more slowly until 12 months, and the mean MV score improved rapidly during the first month after the surgery and then more slowly until 6 months when it reached a plateau. One of the reasons for this discrepancy may be perceptual adaptation, ie, prolonged exposure to visual distortion can alter visual processing within the visual cortex resulting in the brain filtering out distorted stimuli. ${ }^{21-23}$ Another reason may be that the mean $\mathrm{MH}$ and MV scores passed through the score of 0.4 to 0.5 after the 3 months which may be the threshold of perception of metamorphopsia in daily life.

To determine the clinically significant changes in the M-CHARTS score, the reproducibility of M-CHARTS values was examined. A specific criterion for determining the clinically relevant changes in the severity of metamorphopsia is needed to follow the course of disease or to evaluate the effectiveness of the treatments. In addition, it would be helpful for comparing the results by different researchers and for different diseases or treatments. Arimura et al demonstrated that the intra-individual variations of the M-CHARTS score in ERM subjects was within the \pm 0.1 score. ${ }^{3}$ However, some of our patients had relatively greater fluctuations of the M-CHARTS scores. In comparison with the laboratory derived results, the reproducibility of M-CHARTS scores seems to be larger in regular clinical practice where the measurements might be performed by different examiners on different days and at different institutions. Thus, we examined the reproducibility of M-CHARTS scores by determining the $95 \%$ limits of agreement between test and retest measurements by different examiners on different days and at different institutions. The $95 \%$ limit of agreement was within \pm 0.3 $\log \mathrm{MET}$ score for both $\mathrm{MH}$ and MV. The results showed that detecting a clinically relevant change in M-CHARTS score in patients with ERM required a change of $\geq 0.3 \log$ MET score. Although the cases whose metamorphopsia worsened between test and retest were excluded, we cannot eliminate the possibility that the status of ERM might change in some patients between the testing periods. However, we believe that our results are reliable because the disease is relatively stable over a short period.

The ROC analysis showed the best correlation between the changes in the degree of metamorphopsia in daily life and the M2-value. The optimal cutoff value was -0.4 . Taking into account not only the reproducibility but also the consistency with the subjective changes in metamorphopsia, a change of the M2-value by $\geq 0.4$ may be the optimal value for clinically relevant change in the M-CHARTS score after ERM surgery. Using this criterion, the M-CHARTS score improved by $\geq 0.4 \mathrm{M} 2$-value in 46 eyes $(70.8 \%)$, remained unchanged in 14 eyes $(21.5 \%)$, and worsened in five eyes $(7.7 \%)$ at 12 months in Part 1 . A complete resolution in the M-CHARTS scores, ie, both MH and MV scores of 0 , was achieved in $18(27.7 \%)$ patients at 12 months. Bouwens et al demonstrated that the degree of metamorphopsia improved in $82 \%$ of cases and was unchanged in $16 \%$ by using their original metamorphopsia tool, ${ }^{24}$ and Bae et al reported that complete regression of metamorphopsia was achieved in $33.3 \%$ which is comparable to our results. ${ }^{23}$

The optimal cutoff values of raw data of the M-CHARTS including the $\left(\mathrm{MH}_{\text {post }}-\mathrm{MH}_{\text {pre }}\right)$, the $\left(\mathrm{MV}_{\text {post }}-\mathrm{MV}_{\text {pre }}\right)$, and the M1-value were all within the range of reproducibility of the raw data of the M-CHARTS scores, suggesting that these parameters are unsuitable for evaluating the clinically relevant changes in the degree of metamorphopsia (Table 4, Table 5). Thus, evaluating the changes in the M-CHARTS scores in logarithmic form is favorable not only theoretically but also from the perspective of consistency with the subjective changes.

With respect to the correlation between OCT parameters and subjective metamorphopsia in daily life, the CFT and CV in the eyes without metamorphopsia at baseline were significantly smaller than those in the eyes with metamorphopsia at baseline. One of the reasons for this difference may be due to the possibility that the eyes without metamorphopsia at baseline were relatively mild ERM cases. On the other hand, there was no difference in the CFT and CV at baseline and at 12 months between the two groups with and without metamorphopsia at 12 months. These results indicate that CFT and CV are not associated with postoperative metamorphopsia in their daily life.

There are limitations in this study. First, the metamorphopsia in daily life was assessed by a simple questionnaire. Evaluation of metamorphopsia using a more quantitative questionnaire such as the 25-item National Eye Institute Visual Function Questionnaire may demonstrate more 
information on the correlation between the metamorphopsia and the M-CHARTS scores.

\section{Conclusion}

In summary, the resolution rate of subjective metamorphopsia increased as the M-CHARTS scores decreased until finally $35.4 \%$ of patients had no metamorphopsia in their daily life at 12 months after ERM surgery. A complete resolution in the M-CHARTS scores was achieved in $27.7 \%$ of patients at 12 months. When the results of the M-CHARTS are evaluated, a change in the M2-value $\geq 0.4$ may be the optimal criterion for clinically relevant change in metamorphopsia after ERM surgery. Further studies on the M-CHARTS scores especially in patients with other macular diseases would be of value to establish the universal criterion for clinically relevant changes of the M-CHARTS scores.

\section{Disclosure}

The authors have no financial interest or conflicts of interest.

\section{References}

1. Amsler M. Earliest symptoms of disease of macula. Br J Ophthalmol. 1953;37(9):521-537.

2. Matsumoto C, Arimura E, Okuyama S, Tanaka S, Hashimoto S, Shimomura Y. Quantification of metamorphopsia in patients with epiretinal membranes. Invest Ophthalmol Vis Sci. 2003;44(9):4012-4016.

3. Arimura E, Matsumoto C, Okuyama S, Takada S, Hashimoto S, Shimomura Y. Retinal contraction and metamorphopsia scores in eyes with idiopathic epiretinal membrane. Invest Ophthalmol Vis Sci. 2005;46(8):2961-2966.

4. Arimura E, Matsumoto C, Okuyama S, Takada S, Hashimoto S, Shimomura Y. Quantification of metamorphopsia in a macular hole patient using M-CHARTS. Acta Ophthalmol Scand. 2007;85(1):55-59.

5. Okamoto F, Okamoto Y, Hiraoka T, Oshika T. Effect of vitrectomy for epiretinal membrane on visual function and vision-related quality of life. Am J Ophthalmol. 2009;147(5):869-874.

6. Okamoto F, Okamoto Y, Fukuda S, Hiraoka T, Oshilka T. Vision-related quality of life and visual function after vitrectomy for various vitreoretinal disorders. Invest Ophthalmol Vis Sci. 2010;51(2):744-751.

7. Arimura E, Matsumoto C, Nomoto H, et al. Correlations between M-CHARTS and PHP findings and subjective perception of metamorphopsia in patients with macular diseases. Invest Ophthalmol Vis Sci. 2011;52(1):128-135.
8. Ooto S, Hangai M, Takayama K, et al. High-resolution imaging of the photoreceptor layer in epiretinal membrane using adaptive optics scanning laser ophthalmoscopy. Ophthalmology. 2011;118(5):873-881.

9. Kinoshita T, Imaizumi H, OkushibaU, Miyamoto H, Ogino T, Mitamura Y. Time course of changes in metamorphopsia, visual acuity, and OCT parameters after successful epiretinal membrane surgery. Invest Ophthalmol Vis Sci. 2012;53(7):3592-3597.

10. Nowomiejska K, Oleszczuk A, Brzozowska A, et al. M-charts as a tool for quantifying metamorphopsia in age-related macular degeneration treated with the bevacizumab injections. BMC Ophthalmol. 2013;13:13.

11. Kim JH, Kang SW, Kong MG, Ha HS. Assessment of retinal layers and visual rehabilitation after epiretinal membrane removal. Graefes Arch Clin Exp Ophthalmol. 2013;251(4):1055-1064.

12. Roberto dell'Omo, Francesco Cifariello, Ermanno dell'Omo, et al. Influence of retinal vessel printings on metamorphopsia and retinal architectural abnormalities in eyes with idiopathic macular epiretinal membrane. Invest Ophthalmol Vis Sci. 2013;54(12):7803-7811.

13. Ghazi-Nouri SM, Tranos PG, Rubin GS, Adams ZC, Charteris DG. Visual function and quality of life following vitrectomy and epiretinal membrane peel surgery. Br J Ophthalmol. 2006;90(5):559-562.

14. Elliott DB, Sheridan M. The use of accurate visual acuity measurements in clinical anti-cataract formulation trials. Opthal Physiol Opt. 1988;8(4):397-401.

15. Arditi A, Cagenello R. On the statistical reliability of letter-chart visual acuity measurements. Invest Ophthalmol Vis Sci. 1993;34(1): 120-129.

16. Siderov J, Tiu AL. Variability of measurements of visual acuity in a large eye clinic. Acta Ophthalmol Scand. 1999;77(6):673-676.

17. Bland JM, Altman DG. Statistical methods for assessing agreement between two methods of clinical measurement. Lancet. 1986;1(8476):307-310.

18. Akaza E, Fujita K, Shimada H, Yuzawa M. Sighting dominance in patients with macular disease. Nippon Ganka Gakkai Zasshi. 2007; 111(4):322-325.

19. Wong JG, Sachdev N, Beaumont PE, Chang AA. Visual outcomes following vitrectomy and peeling of epiretinal membrane. Clin Experiment Ophthalmol. 2005;33(4):373-378.

20. Bouwens MD, Jong FD, Mulder P, Van Meurs JC. Results of macular pucker surgery: 1- and 5-year follow-up. Graefes Arch Clin Exp Ophthalmol. 2008;246(12):1693-1697.

21. Webster MA, MacLin OH. Figural aftereffects in the perception of faces. Psychon Bull Rev. 1999;6(4):647-653.

22. Gheorghiu E, Kingdom FA. Spatial properties of curvature-encoding mechanisms revealed through the shape-frequency and shape-amplitude sfter-effects. Vis Res. 2008;48(9):1107-1124.

23. Bae SH, Kim D, Park TK, Han JR, Kim H, Nam W. Preferential hyperacuity perimeter and prognostic factors for metamorphopsia after idiopathic epiretinal membrane surgery. Am J Ophthalmol. 2013;155(1):109-117.

24. Bouwens MD, Van Meurs JC. Sine Amsler Charts: a new method for the follow-up of metamorphopsia in patients undergoing macular pucker surgery. Graefes Arch Clin Exp Ophthalmol. 2003;241(2):89-93.
Clinical Ophthalmology

\section{Publish your work in this journal}

Clinical Ophthalmology is an international, peer-reviewed journal covering all subspecialties within ophthalmology. Key topics include: Optometry; Visual science; Pharmacology and drug therapy in eye diseases; Basic Sciences; Primary and Secondary eye care; Patient Safety and Quality of Care Improvements. This journal is indexed on Submit your manuscript here: http://www.dovepress.com/clinical-ophthalmology-journal

\section{Dovepress}

PubMed Central and CAS, and is the official journal of The Society of Clinical Ophthalmology (SCO). The manuscript management system is completely online and includes a very quick and fair peer-review system, which is all easy to use. Visit http://www.dovepress.com/ testimonials.php to read real quotes from published authors. 